\title{
Quality provision under conditions of oligopoly
}

\author{
Johan Willner $^{1}$ (D) Sonja Grönblom ${ }^{1}$
}

Received: 21 February 2019/Accepted: 20 July 2020/Published online: 10 August 2020

(C) The Author(s) 2020

\begin{abstract}
We analyse a market where quality is reflected in sunk costs and/or marginal costs. Firms provide too low quality as compared to the socially optimal solution whenever quality affects (at least) sunk costs. Entry would then increase welfare, but the number of firms is restricted by an upper limit that depends on how sunk costs and consumer utility are affected by a quality change. Firms may even produce an excessive output if both types of costs are dependent on quality. Moreover, entry reduces quality except for when the number of firms increases from two to three. Quality is on the other hand socially optimal and independent of market structure if only marginal costs are affected by quality, but output is too low unless the number of firms is very high.
\end{abstract}

Keywords Product quality · Oligopoly $\cdot$ Competition

JEL Classification L33 · D21 · D43 · H42

\section{Introduction}

We are used to associate imperfect competition with too low consumption and hence a welfare loss. However, welfare also depends on product quality, and we shall show that a symmetric oligopoly produces too low quality if quality is reflected in sunk costs. This distortion can (under some circumstances) overshadow the traditional deadweight loss of imperfect competition. Moreover, entry will worsen the quality distortion (except for when a third firm enters in a duopoly). While these results do not apply when quality affects (only) marginal costs, their presence in the opposite case represents a sufficient reason to challenge conventional views on the best ways to organise an industry.

Johan Willner

jwillner@abo.fi

1 Department of Economics, Åbo Akademi University, Fabriksgatan 2 and Fänriksgatan 3, 20500 Turku, Finland 
It is well known that quality can be either too high or too low (relative to the socially optimal solution) in a profit-maximising monopoly, depending on how quality affects the slope of inverse demand (Spence 1975; Sheshinski 1976). The literature on spatial models of horizontal product differentiation suggests that quality may be a matter of concern under oligopoly as well. ${ }^{1}$ Cases in point are Ma and Burgess (1993) (who assume that firms decide on quality and price sequentially) and Brekke et al. (2010) (who focus on simultaneous decisions). Models with endogenous entry suggest that the market may also provide too many varieties in addition to suboptimal quality (Economides 1991). ${ }^{2}$ However, less is known about quality and the impact of competition without the additional market imperfection of product differentiation. Our analysis therefore fills a gap.

When it comes to the amount of competition, we conform to most of the earlier literature by focusing on the number of firms. While an increase in the number of firms tends to increase quality if it reduces the quality-related fixed costs, its impact seems otherwise ambiguous (Banker et al. 1998). ${ }^{3}$ It has also been argued that entry might reduce welfare by narrowing the quality spectrum when firms produce several quality levels under quantity competition and uniformly distributed tastes (Gal-Or 1983). However, increased competition can also mean lower transportation costs (with an ambiguous effect on quality) or higher firm density (with a non-negative impact) in Brekke et al. (2010). The amount of competition has also been related to the size of the market, not least in the case of economic integration. While this interpretation has been more common in the literature on innovations and growth (Vives 2008; Rivera Batiz and Romer 1991; Berry and Waldfogel 2010) find that a larger market can increase product quality as well. We shall also present some results that apply to this interpretation.

Quality is likely to depend on product design and other factors that are associated with sunk costs. We therefore develop a model of fully informed consumers by Sutton (1991), who also assumes that goods are potentially differentiated but homogeneous ex-post. ${ }^{4}$ It is well known that there is then an upper limit for the number of firms that can break even. This maximum number is independent of the market size, and low under reasonable conditions. To make the model more useful for dealing with entry and near-perfect competition we relax Sutton's assumption

\footnotetext{
${ }^{1}$ It has also been shown that moral hazard, adverse selection, or signalling may cause quality to be either under- or overprovided (Tirole 1988; Belleflamme and Peitz 2014; Kranton 2003), but such issues are outside our scope.

2 Regulation may have a positive impact under such conditions (Ma and Burgess 1993; Economides 1991), but quality can also become too high, and firm density too low (Brekke et al., 2006). Regulated pricing below the competitive level can also encourage differently located duopolists to increased quality in the form of reduced congestion (Matsumura and Matsushima 2007). The impact of competition on quality can on the other hand become positive if producers are partly altruistic (Brekke et al., 2011).

3 Increased competition can also mean reduced dominance in a duopoly (in which case the impact on quality depends on which firm that has a cost advantage) and reduced cooperation (in which case the outcome depends on the existence of synergistic benefits) in Banker et al. (1998).

4 Sutton's model was applied on branding, i.e. perceived quality, rather than on quality as such, but the logic is similar. As for the notion of an ex-post symmetric equilibrium with fully informed consumers and potentially low quality, we refer to the US market for bulk beer: 85 per cent of the market consists of bland brands that are hard to distinguish (Choi and Stack 2005).
} 
that consumption and quality have the same impact on utility. A high maximum number is then likely to mean that there is a scope for entry if enterprise formation is sluggish, as argued by Geroski (1995). We also extend Sutton's analysis by assessing social optimality, and by including the case where quality is reflected in marginal costs.

We show that quality is lower than in the first best solution and decreasing in the number of firms (except for in a duopoly) if it affects sunk costs or both sunk costs and marginal costs. It also follows that an oligopoly can under some circumstances be associated with an excessive output of cheap low-quality goods. An increase in the size of the market will on the other hand lead to higher quality, even without entry. However, there is no quality distortion (and no limit to the number of firms) if quality affects only marginal costs. While output is then too low if firms are few, the allocation approaches a textbook-model of perfect competition when their number becomes sufficiently large.

We introduce a couple of extensions in order to assess robustness. Results become roughly similar with simultaneous instead of sequential decision-making (and we explain why there is a difference as compared to the earlier literature). We also extend the analysis to quasilinear utility, which allows us to relax the assumption that a uniform increase in quality has no impact on the willingness to pay. Results are also then roughly similar, but there is not always any upper limit to the number of firms.

We proceed as follows. The basic components of the model (consumers, the $n$ firm oligopoly and the socially optimal solution) are presented in Sect. 2. In Sect. 3 we evaluate the performance of the $n$-firm oligopoly (as compared to the socially optimal solution) and analyse the comparative statics of entry (within the feasible range) and market size. In Sect. 4 we extend the analysis to simultaneous maximisation and to quasilinear utility. Section 5 presents some concluding remarks. Proofs and other technical details are included in an appendix with sections "Appendix 1-13".

\section{The model}

\subsection{Costs and utility}

Consider a market where each producer $(i)$ has to decide on both quality $\left(q_{i}\right)$ and output $\left(y_{i}\right)$. Firm $i$ has an advantage over firm $j$ if $q_{i}>q_{j}$, but higher quality also means higher costs. In what follows, we refer to the case where quality is reflected in sunk costs $\left(S_{i}\right)$, for example as related to product design or $\mathrm{R} \& \mathrm{D}$, but not in marginal costs, as case (a). Other sunk are ruled out. The case where quality affects only marginal costs $\left(c_{i}\right)$, for example via more expensive inputs or more timeconsuming procedures will be referred to as case $(b)$. These cases yield explicit solutions for output and quality. Quality may however also affect both types of costs. We refer to this situation as case (c). Some important conclusions can be extended to this case as well.

The cost function for the more general case (c) is 


$$
T_{i}=c_{i} y_{i}+S_{i}=\left(c_{0}+q_{i}^{\gamma}\right) y_{i}+s q_{i}^{\beta},
$$

where $c_{0}, \gamma, \beta$, and $s$ stand for positive parameters. To get the cost function for case (a), we set $\gamma=0$ and $s>0$ (in which case $c_{i}=c_{0}+1$ for all (i). Setting $\gamma>0$ and $s=0$ yields case (b). ${ }^{5}$ Note that $\beta$ represents the elasticity of sunk costs with respect to quality, or shortly the sunk cost elasticity. This parameter has a crucial impact on the maximum number of firms. Note also that the cost function implies constant marginal costs (with respect to output), and a positive cross derivative with respect to quality and output (i.e. cost substitutability between quality and quantity; see Brekke et al. 2010).

As for the demand side, there are $N$ identical consumers with the income $m$. Consider the utility function of an individual $h, h=1,2, \ldots N$, who consumes the output of producer $i$ :

$$
u\left(y_{h i}, q_{i}, z_{h}\right)=y_{h i}^{\alpha_{1}} q_{i}^{\alpha_{2}} z_{h}^{\alpha_{0}},
$$

where $z_{h}$ stands for a basket of other goods with a unit price. The exponents $\alpha_{1}, \alpha_{2}$, and $\alpha_{0}$ stand for the elasticity of utility with respect to the consumption of $y$, to its quality, and to the consumption of $z$.

We assume without loss of generality that $\alpha_{0}=1-\alpha_{1}$. Note that $\alpha_{1}=\alpha_{2}$ in Sutton's version of the model (Sutton 1991). However, our analysis also includes the cases of utility being either more or less sensitive to the quality than to the quantity of $y$, i.e. that either $\alpha_{2} / \alpha_{1}>1$ or $\alpha_{2} / \alpha_{1}<1$ holds true. While $\alpha_{1}$ determines the size of the industry revenues $R=\alpha_{1} m N$, in most cases the utility function parameters matter only via the ratio $\alpha_{2} / \alpha_{1}$, which will therefore be abbreviated as $\rho$. For example, we can maximise $q^{\rho} y$ instead of (2) when deriving the socially optimal solution. Note also that (2) implies that the inverse market demand is $p=\alpha_{1} m N /$ $y=R / y$; for more details, see "Appendix 1 ".

We focus on a subgame perfect Cournot-Nash equilibrium with sequential decisions on market participation, quality, and output (except for in Sect. 4). We first analyse how firm 1 maximises profits with respect to $y_{1}$ given all $q_{i}$ (with no loss of generality), and thereafter how this firm's maximum profits $\pi_{1}$ are maximised with respect to $q_{1}$, given its competitors' quality levels. The market solution is obtained after imposing ex-post symmetry. Finally, we analyse the zeroprofit condition in order to understand the range of feasible market structures. Details are presented in "Appendix 2".

However, our simplifying assumption of Cobb-Douglas utility has a couple of less desirable implications. First, there is no impact on the willingness to pay if all firms increase their quality in the same way (although there is a positive impact on firm $i$ 's residual demand when $q_{i}>q_{j}$ ). The possibilities of higher or lower demand because of higher quality among all producers are thereby ignored. ${ }^{6}$ While changing

\footnotetext{
${ }^{5}$ Note that we have ruled out sunk costs that are not related to quality, so $s>0$ and $\beta=0$ cannot hold true at the same time.

${ }^{6}$ Demand may become lower because of higher quality if this reduces the need for replacements. This mechanism explains why several firms (including Philips, Osram, and General Electric) agreed to increase their sales by reducing the life-span of their light bulbs by approximately a half in 1924 (MacKinnon, 2016).
} 
the quality of US-style bulk beer might not necessarily change drinking habits, the assumption of no impact is in many other cases likely to be too restrictive. Second, the fact that (1) implies unitary demand elasticity rules out an equilibrium under unregulated (profit-maximising) monopoly. ${ }^{7}$ We therefore demonstrate the robustness of our main results by including an extension where these two limitations do not apply.

The following technical assumption ensures that at least two firms can break even in case (a), that there is a meaningful oligopoly solution in case (b), and a meaningful social optimum in case (c):

Assumption 1 (i) $\beta / \rho \geq 1$; (ii) $\gamma>\rho$; (iii) $\beta>\gamma-\rho$.

It will be convenient to abbreviate $\beta / \rho$ as $\varepsilon$, not least because of its impact on the maximum number of firms. We cannot assess the impact of entry unless $\varepsilon \geq 8 / 3$, so that at least three firms can break even. It will also be convenient to abbreviate $\gamma / \rho$ as $\eta$.

\subsection{The oligopolistic equilibrium}

Denote the price charged by firm $i$ by $p_{i} ; p$ stands for the price in a symmetric equilibrium. Intuitively, several quality levels cannot co-exist unless all firms have the same quality-to-price ratio. The insight that $q_{i}^{\rho} / p_{i}=q_{j}^{\rho} / p_{j}$ will be useful for deriving the inverse demand function for a given firm and is therefore explained in "Appendix 1".

Focusing on firm 1 , the fact that $q_{i}^{\rho} / p_{i}=q_{j}^{\rho} / p_{j}$ implies that $p_{j}=p_{1}\left(q_{j} / q_{1}\right) \rho$ for all $j=2,3, \ldots n$ under ex ante product differentiation. The industry sales revenues are therefore:

$$
R=y_{1} p_{1}+p_{1} \sum_{j=2}^{n} y_{j}\left(\frac{q_{j}}{q_{1}}\right)^{\rho} \text {. }
$$

Rearrange to get the inverse demand function for firm 1:

$$
p_{1}=\frac{R}{y_{1}+\sum_{j=2}^{n} y_{j}\left(q_{j} / q_{1}\right)^{\rho}} .
$$

The profit function is therefore:

$$
\pi_{1}=\frac{R}{y_{1}+\sum_{j=2}^{n} y_{j}\left(q_{j} / q_{1}\right)^{\rho}} y_{1}-\left(c_{0}+q_{1}^{\gamma}\right) y_{1}-s q_{1}^{\beta} .
$$

As shown in "Appendix 2", a unique and meaningful solution exists when Assumption 1 is satisfied. Consider the equilibrium output and quality in case (a), where $\gamma=0$ and $s>0$ :

\footnotetext{
${ }^{7}$ Firms may of course be regulated, or they may not be profit maximisers, but consumers are otherwise at the mercy of the producers whenever the absolute value of the price elasticity of demand is lower or equal to $1 / n$.
} 


$$
\begin{gathered}
y^{C}=\frac{R(n-1)}{c n} \\
q^{C}=\left[\frac{2 \rho R(n-1)^{2}}{s \beta n^{3}}\right]^{1 / \beta}=\left[\frac{2 R(n-1)^{2}}{s \varepsilon n^{3}}\right]^{1 / \beta} .
\end{gathered}
$$

The preference for quality as represented by $\rho$ affects $q^{C}$ but not $y^{C}$, which is the same as in a conventional Cournot-model with a fixed quality (see however Sect. 4.2). The same applies to the price $p^{C}=c n /(n+1)$.

Next, consider the solution for case (b) where $s=0$ and $\gamma>0$ :

$$
\begin{gathered}
y^{C}=\frac{(n-1)(\gamma-\rho) R}{n \gamma c_{0}}=\frac{(n-1)(\eta-1) R}{n \eta c_{0}}, \\
q^{C}=\left[\frac{\rho c_{0}}{\gamma-\rho}\right]^{1 / \gamma}=\left[\frac{\eta c_{0}}{\eta-1}\right]^{1 / \gamma} .
\end{gathered}
$$

In this case, a higher preference for quality (i.e. a higher value of $\rho$ ) results in a lower output and a higher price $\left(p^{C}=n \gamma c_{0} /(n-1)(\gamma-\rho)=n \eta c_{0} /(n-1)(\eta-1)\right)$.

\subsection{The socially optimal solution}

The socially optimal solution provides a reference point when asking whether the quality becomes too high or too low in an oligopoly, but it can also be interpreted as the outcome of welfare maximisation, for example in the case of competent an unbiased ideal public ownership. Such an optimum includes just one quality level, because product differentiation would be pointless when all consumers are identical.

It is well known that maximisation of the total surplus would cause a deficit in the presence of exogenous sunk costs. To avoid extending the analysis to the potentially distortionary taxes that the deficit calls for, it is convenient to impose a break-even constraint. We would then get the same result with the simpler procedure of constrained maximisation of the consumer surplus (or even output). The case of endogenous sunk costs is somewhat more complicated. Maximisation of the total surplus can then yield positive profits. ${ }^{8}$ This would potentially involve stakeholders external to the industry, like in the case of a deficit. To avoid such discussions we maximise the consumer benefits (in which case the break-even constraint is always binding).

Consider the welfare of a typical consumer:

\footnotetext{
${ }^{8}$ It can easily be verified that maximisation of the total surplus can yield non-negative profits in case a). Suppose that $c_{0}=1, s=1, \alpha_{1}=\alpha_{2}=\alpha_{0}=0.5$, and note that this implies that $N u(y / N, q, z)=(y q R)^{0.5}$. Maximising $(y q R)^{0.5}+R-y-q^{\beta}$ then yields a solution that is consistent with nonnegative profits unless $R>6.6251 \times 10^{9}$. Also, the profits would be higher than the consumer benefits unless $R>621,161.06$. As for the more general case c), see the discussion after Proposition 2 in Sect. 3.
} 


$$
u\left(y_{h}, q, z\right)=\left(\frac{y}{N}\right)^{\alpha_{1}} q^{\alpha_{2}} z^{\alpha_{0}} .
$$

To get the socially optimal solution, solve for $y$ from the break-even constraint, insert the solution into (10), and maximise with respect to $q$ (see "Appendix 3"). Setting $\gamma=0$ then yields:

$$
\begin{gathered}
y^{G}=\frac{\beta R}{(\rho+\beta) c}=\frac{\varepsilon R}{(1+\varepsilon) c} \\
q^{G}=\left[\frac{\rho R}{(\rho+\beta) s}\right]^{1 / \beta}=\left[\frac{R}{(1+\varepsilon) s}\right]^{1 / \beta} .
\end{gathered}
$$

It follows that the price is $(1+1 / \varepsilon) c$.

As for cost functions of type (b), we get marginal-cost pricing both with unconstrained maximisation of the total surplus and with constrained maximisation of the consumer surplus:

$$
\begin{gathered}
y^{G}=\frac{(\gamma-\rho) R}{\gamma c_{0}}=\frac{(\eta-1) R}{\eta c_{0}}, \\
q^{G}=\left(\frac{\rho c_{0}}{\gamma-\rho}\right)^{1 / \gamma}=\left(\frac{c_{0}}{\eta-1}\right)^{1 / \gamma} .
\end{gathered}
$$

The price is then $\gamma c_{0} /(\gamma-\rho)$. "Appendix 4 " includes a discussion of how the solution is affected by changes in $R, s, \beta, \rho$, and $\gamma$.

\section{The performance of the $n$-firm oligopoly}

\subsection{The maximum number of firms}

It is well known that endogenous sunk costs related to quality or an image of quality can limit the number of firms irrespective of the market size (Sutton 1991). We extend this insight to the cases of $\alpha_{1} \neq \alpha_{2}$ and $s \neq 1$ :

Lemma 1 The following holds true in case (a): (i) The feasible range of oligopolistic market structures is represented by numbers of firms such that

$$
n \leq \hat{n}=1+\frac{\beta}{4 \rho}+\sqrt{\frac{\beta^{2}}{16 \rho^{2}}+\frac{\beta}{2 \rho}}=1+\frac{\varepsilon}{4}+\sqrt{\frac{\varepsilon^{2}}{16}+\frac{\varepsilon}{2}} .
$$

(ii) This upper limit is increasing in the sunk-cost parameter $(\beta)$ but decreasing in the quality elasticity $(\rho)$, and independent of the market size $(R)$.

(iii) There is no upper limit to the number of firms in case (b).

Proof See “Appendix 5". 
The intuition for why $\hat{n}$ increases with $\beta$ is explained by the fact that steeply increasing sunk cost discourage firms from investing in quality. The scope for entry increases because of the low quality-related sunk costs. Assumption 1(i) ensures that at least two firms can break even, but it is not meaningful to analyse entry unless $\varepsilon \geq 8 / 3$, so that $n$ can increase, at least from two to three. The fact that $\rho<1$ can hold true (so that quality can have a weaker impact on consumer utility than quantity) implies that more firms than in Sutton (1991) can break even (and vice versa if $\rho>1$ ). For example, suppose that $\rho=1$ and $\beta=6.4$, so that five firms can break even. Changing $\rho$ to 0.0666 (or changing $\beta$ to 48.048 and $\rho$ to 0.5 ) would mean $\varepsilon=96.096$ and hence $\hat{n}=50$. Intuitively, a weak preference for quality relative to quantity means a low incentive for firms to increase their quality-related sunk costs $s\left[q^{C}\right]^{\beta}$ and vice versa, as also follows from (7).

According to part (ii), $\hat{n}$ is independent of the market size in case (a), because relaxing the assumption $\alpha_{1}=\alpha_{2}$ does not change the fact that net variable costs, sunk costs, and hence net profits, are all directly proportional to $R$. However, this does not apply to case (c), because marginal costs are $c_{0}+q^{\gamma}$. As for (iii), there are no sunk costs, so profits remain nonnegative but approach zero when $n$ approaches infinity.

\subsection{Entry and changes in the size of the market}

The market may be highly concentrated if $\varepsilon$ is low (see Lemma 1). However, there may be few firms despite a high value of $\varepsilon$ if enterprise formation is sluggish, as suggested by Geroski (1995). This would imply a scope for entry and for an impact of the market size, but the analysis also raises the question about whether entry is desirable.

Proposition 1 The following holds true for the n-firm oligopoly: In cases (a) and (c), quality is increasing in $R$, and decreasing in $n$ except for when $n$ increases from 2 to 3. In case (b), quality is independent of both $R$ and $n$.

Proof See "Appendix 6".

The impact of the size of the market is thus positive, as suggested by the empirical findings in Berry and Waldfogel (2010). The intuition for the results in cases (a) and (c) is based on the higher ability to afford quality-enhancing fixed costs.

There is a similarity between the result that the highest quality is achieved when $n=3$ and models of innovation and growth in the spirit of Schumpeter, where the highest growth is achieved in an oligopoly (see Carlin and Soskice 2006). To understand the intuition, note that producers are not rewarded for quality as such. The inverse demand function remains $p=R / y$ if all firms double their quality. Higher quality in the industry would therefore not increase a firm's gross profits (the difference between total revenues and variable costs), but its sunk costs would be higher. ${ }^{9}$ A firm is on the other hand rewarded through higher gross profits if its

\footnotetext{
${ }^{9}$ Higher quality works technically in the same way as when a utility function is multiplied by a positive constant: the number assigned to the utility level changes, but there is no change in consumer choice.
} 
quality is higher than among its competitors. However, this increment is decreasing in both quality and the number of firms. It has to be weighted against the increment in sunk costs, which is increasing in quality and independent of the number of firms. These increments are equal in equilibrium. The industry gross profits $(R / n)$ are inversely related to the number of firms, and a relative change in quality just redistributes profits between different firms. It is easy to understand why the equilibrium quality falls when $n \geq 3$, as the rewards to superior quality are shifted downwards after entry. However, the opposite holds true when a third firm enters in a duopoly, because the reduction in industry gross profits is not significant enough to offset the gains related to the higher market share that is caused by.

As for case (b), the increment in the gross profits is shifted in the same way as in case (a) and is highest for a given quality level for $n=3$. However, this increment does not have to be balanced against any increment in sunk costs. Moreover, its maximum does not depend on the number of firms.

Our analysis can also be applied on the simultaneous increase in market size and the number of competitors that would be associated with economic integration. For example, suppose that the markets of two countries indexed by 1 and 2 are joined, thus creating a market of the size $R_{1}+R_{2}$ and the number $n_{1}+n_{2}$ of firms. It is obvious from Proposition 1 that this leads to a higher output. As for quality, there is no trade-off between quality and output in case (b), so we focus on case (a). Consider an industry with an initial market size $R_{0}$ and with $n_{0}$ firms. Integration (with an arbitrary number of economies) then makes the industry part of a common market where $R>R_{0}$ : and where $n \geq n_{0}$ :

Corollary 1 Suppose that quality affects sunk costs only and consider increases by $\Delta R$ and $\Delta n$ in $R$ and $n$, assuming that $n_{O}+\Delta n<\hat{n}$. (i) The condition for quality to increase is $d R / R_{O}>\left[\left(n_{0}-3\right) /\left(n_{0}-1\right)\right] d n / n_{0}$; (ii) An equiproportional increase in $n$ and $R$ leads to higher quality;(iii) Integrating economies where $\varepsilon$ is equal and where $n=\hat{n}$ leads to higher quality.

\section{Proof See "Appendix 7".}

Parts (i) and (ii) suggest that the integration of a number of identical industries would increase quality. This implies by continuity that there is scope for a quality improvement if the industries are sufficiently similar. Intuitively, the positive impact of a larger market compensates for the negative impact of an increase in the number of firms. On the other hand, part (i) implies that quality might deteriorate if the markets are too heterogeneous. This may happen if the relative increase in market size is moderate as compared to the relative increase in the number of firms. Suppose for example that there are 11 firms in Country 1, and 14 firms in Country 2. Also, suppose that all other parameters including $R$ are equal, and that all firms are able to break even also in the common market. The citizens in Country 1 will then experience a lower quality. 


\subsection{Welfare implications}

Low quality is not necessarily the same as low welfare, so we need to establish the relationship between market provision and the socially optimal solution as defined in Sect. 2.3.

Proposition 2 (i) Consumer welfare is increasing in $n$ and $R$ in the feasible interval in cases (a) and (b). (ii) The quality is below its socially optimal level in cases (a) and (c), but quality is too low or too high in case (b). (iii) Output is otherwise below its socially optimal level in cases $(a)$ and $(b)$, but equal to the socially optimal level when $n=2$ and $\varepsilon=1$ in case (a), and either below or above this level in case (c).

\section{Proof See "Appendix 8".}

Part (i) also suggests that an increase in both market size and the number of firms, as for example caused by economic integration, improves welfare. As for part (ii), quality is too low in cases (a) and (c) because of the absence of any demand shift that would compensate for the higher sunk costs (see however Sect. 4.2). While higher quality gives a firm an advantage over its competitors, entry reduces its ability to afford higher sunk costs. Social optimality is on the other hand defined in terms of utility, which is increases with $q$ even if a higher $q$ does not cause a demand shift. Moreover, profits are zero, and the absence of competition means that there is no duplication of sunk costs, so society can afford a higher quality. As for case (b), the quality provided by the market is socially optimal. This means that the market approaches the socially optimal solution when $n$ and hence $y$ increases.

As for part (iii), we are used to think of imperfect competition as restricting output. However, a duopoly provides the socially optimal output in case (a) when $\varepsilon=1$. This happens because the product becomes sufficiently cheap because of its suboptimal quality. ${ }^{10}$ Moreover, low quality can in case (c) be associated with even higher consumption than under social optimality. Both types of costs are then strongly sensitive to quality. While it is easier to find parameter values that generate the opposite outcome, the following example shows that we cannot rule out an excessive output. Consider the following parameter values: $\alpha_{1}=\alpha_{0}=0.5, \alpha_{2}=1.5$, $m=0.045, N=10,000, c_{0}=0.12, \beta=14, \gamma=3, s=0.24$, and $n=7$. These values imply $R=225$ and $\rho=3$. The oligopoly would then yield $y^{C}=134.7853$ and $q^{C}=1.0944$, as compared to the outcome of maximising consumer utility subject to a break-even constraint, which would yield $y^{G}=120.8558$ and $q^{G}=1.2002$. The prices would be $p^{C}=1.8141$ and $p^{G}=1.8617$.

This example also highlights the fact that the break-even constraint is not necessarily binding when maximising the total surplus $(T S) .{ }^{11}$ Such a procedure would yield $T S^{G}=252.3736$ (as compared to $T S^{C}=232.8438$ in the oligopoly). We would also get $y^{G}=35.5425$ and $q^{G}=1.1002$, which makes the oligopolistic overproduction of a low-quality good appear as even more pronounced. However, as

\footnotetext{
${ }^{10}$ Note however that Lemma 1 implies that there is no scope for entry under such conditions.

${ }^{11}$ Note that the total surplus is $N u+\Sigma_{i} \pi_{i}$ in the oligopoly but $N u+\pi$ when we consider social optimality.
} 
pointed out in Sect. 2.3, the choice of maximand can be problematic when sunk costs are quality-related. Maximising $T S$ would yield even higher profits than in the oligopoly $\left(\pi^{G}=149.1724\right.$, in contrast to $\pi^{C}=41.5686$ and $\left.\pi_{i}^{C}=5.9384\right)$. The highest consumer benefits that yield zero profits would on the other hand yield a lower total surplus (216.7957) than in the oligopoly. ${ }^{12}$

While entry would cause the market to approach the socially optimal solution in case (b), Propositions 1 and 2 also suggest that there is a trade-off in case (a): entry can increase welfare by causing output to be higher, but only at the expense worsening the quality-related distortion. ${ }^{13}$ This prevents the market from converging towards the socially optimal solution even when $\hat{n}$ is large.

Case (a) also highlights the fact that higher costs are not as such signs of inefficient production. Costs in the oligopoly may deviate from social optimality in both directions despite identical production functions:

Corollary 2 (i) Marginal costs are higher in the socially optimal allocation than in the oligopoly in case (c), but equal in cases (a) and (b). (ii) Unit costs are the same in the oligopoly as in the socially optimal solution when $n=2$ in case (a), but they are otherwise higher and increasing in $n$. There is no difference in unit costs in case (b), but the comparison is ambiguous in case (c).

\section{Proof See "Appendix 9".}

Intuitively, there are two necessary conditions for marginal costs to be higher in the socially optimal allocation: they have to depend on quality like in cases (b) and (c), and quality has to be higher in the socially optimal solution, like in case (a). As for the unit costs when $n>2$ in case (a), note that entry reduces both quality-related sunk costs and output in each firm, but the sunk costs per unit of output is increasing in $n$, as follows from (6) and (7). In case (c), the ambiguity depends on the fact that marginal costs and average sunk costs are likely to differ from the socially optimal solution in opposite directions.

\section{Extensions}

\subsection{Simultaneous maximisation}

Decisions about R\&D- and other investments often have to be made before deciding about output, so it makes sense to assume two-stage maximisation. However, we shall demonstrate that our main results are valid also in the case of simultaneous maximisation. By being analytically simpler, this case might also make it easier to understand the basic mechanisms at work.

\footnotetext{
${ }^{12}$ We may also strike a compromise between the approaches. Suppose that we maximise consumer utility subject to the constraint that the profits are 38 . This would yield $T C^{G}=234.7301$, which is higher than in the oligopoly. As compared to the oligopoly, output is lower but quality higher $\left(y^{G}=102.6639\right.$ and $q^{G}=1.1881$ ).

${ }^{13}$ Note also that differentiating (7) with respect to $\beta$ shows that quality has a minimum for $\beta=2 R(n-1) 2 e \rho / s n^{3}$. Like in the case of the socially optimal solution, quality may become increasing in $\beta$ when $\beta$ becomes sufficiently high.
} 
We limit our attention to cost functions of type (a) and (b). The socially optimal solution is then same as in the two-stage version. As for the $n$-firm oligopoly in case (a), differentiate (5) with respect to both $y_{1}$ and $q_{1}$ before imposing symmetry. This yields the same industry output as before, i.e. (6). However, the socially optimal quality is in most cases different in case (a). The first-order condition then becomes:

$$
-\frac{R y_{1}}{\left[y_{1}+\sum_{j=2}^{n} y_{j}\left(q_{j} / q_{1}\right)^{\rho}\right]^{2}}\left(-\frac{\rho \sum_{j=2}^{n} y_{j} q_{j}^{\rho}}{q_{1}^{1+\rho}}\right)-s \beta q_{1}^{\beta-1}=0 .
$$

Impose ex-post symmetry and solve for $q$ :

$$
q(n)=\left[\frac{\rho R(n-1)}{s \beta n^{2}}\right]^{1 / \beta}=\left[\frac{R(n-1)}{s \varepsilon n^{2}}\right]^{1 / \beta} .
$$

Like in the case of two-stage maximisation, there is a limit, here denoted by $n^{S}$, to the number of firms. Also, this limit does not depend on $R$. Impose symmetry on (5) and insert (17). It follows that firms can break even only if $n \leq n^{S}=1+\varepsilon$. It follows from ("Appendix 10") in the proof of Proposition 2(iii) in "Appendix 9" that $\hat{n}$ (as defined by Lemma 1 ) is the same if $\varepsilon=1$, so that $n^{S}=\hat{n}=2$, but lower than $n^{S}$ for higher values of $\varepsilon$.

As for case (b), maximise (5) with respect to $y_{1}$ and $q_{1}$ when $s=0$, impose symmetry and solve for $q$ and $y$. We then get the same solution as under two-stage maximisation.

Comparing Proposition 3 below to Proposition 2 shows that the results of simultaneous maximisation are similar to the results in Sect. 3, but a few details differ:

Proposition 3 The following results apply to simultaneous maximisation in an oligopoly: (i) Quality is too low and decreasing in the number of firms in case (a), but socially optimal and independent of the number of firms in case (b); (ii) Quality is the same as under two-stage maximisation in case (a) if $n=2$, but otherwise lower; (iii) Output is socially optimal if $n=n^{S}=1+\varepsilon$ in case a,) but otherwise lower.

\section{Proof See "Appendix 10".}

The results from Sect. 3 are in other words fairly robust when it comes to the stage at which quality is chosen. As for the intuition, it is similar when it comes to part (i). However, the lower quality under simultaneous maximisation in part (ii) differs from the findings of Ma and Burgess (1993), who assume exogenous location. To understand why this is the case, note that simultaneous decisions and Bertrand competition would mean treating other firms' prices as constants when choosing $p_{i}$ and $q_{i}$. Sequential decision-making would on the other hand cause each firm to take into consideration the fact that its competitors react to its quality increase by reducing their prices. This reduces a firm's incentive to provide high quality. We predict a higher quality in the Cournot case because each firm treats its competitors' outputs as constants when $p_{i}$ and $q_{i}$ are chosen simultaneously (as 
reflected in $\left.\partial \pi_{i} / \partial q_{i}\right)$. The only benefit of a relatively higher quality is then the positive direct effect on its residual demand, as reflected in a reduced denominator in the first term of (5). Sequential decision making would on the other hand mean that the firm can take into consideration the fact that its competitors would respond by producing less. This strengthens its incentive to provide quality. As for (iii), output is socially optimal when $n=1+\varepsilon$. However, in contrast to two-stage maximisation, which implies $\hat{n} \leq n^{S}=1+\varepsilon$, this does not necessarily mean a duopoly. More firms can now break even because of a lower equilibrium quality.

Some other observations can also be made. Consumer welfare is increasing in the number of firms also under simultaneous maximisation. In case (a), this follows from (6) and (17) if $n<3+\varepsilon$, so the result applies whenever firms can break even, i.e. when $n \leq n^{S}=1+\varepsilon$. In case (b), welfare increases because output is increasing in $n$ while quality remains unchanged. As for unit costs in case (a), they are now $c(1+1 / \varepsilon)$ both in the oligopoly and in the socially optimal solution. The fact that they do not become higher than in the socially optimal solution (contrary to Corollary 2) is explained by lower quality.

\subsection{The case where quality increases the willingness to pay}

While otherwise attractive, the utility function in Sects. 2, 3 rules out any impact on demand of a uniform quality increase across all firms. To demonstrate the robustness of our main findings despite such a restrictive assumption, we explore a modified version of a model in Ritz (2014). ${ }^{14}$ We can then also relax the assumption that $n \geq 2$, but income effects are now ignored, and we have to assume that outputs of different quality can be added. ${ }^{15}$

The notation from Sects. 2, 3 is otherwise unchanged, but we focus on a representative consumer with quasilinear utility with the positive parameters $a, b$, and $k^{16}$ :

$$
u=a \sum_{i=1}^{n} y_{i}+k \sum_{i=1}^{n} q_{i} y_{i}-\frac{b}{2}\left(\sum_{i=1}^{n} y_{i}\right)^{2}+z
$$

The cost function is the same as (1), but $s=0.5$ and $\beta=2$ in case (a), and we replace $q^{\gamma}$ by $0.5 q^{2}$ in case (b). To ensure a meaningful solution for all $n$ we assume $2 b>k^{2}$.

Budget balance requires $\sum_{i=1}^{n} p_{i} y_{i}+y_{0}=m$. Replace $z$ by $m-\sum_{i=1}^{n} p_{i} y_{i}$ in (18), maximise with respect to $y_{i}$, and rearrange to get the following inverse demand function:

\footnotetext{
14 Ritz' model was originally applied on imperfectly competitive welfare losses. See also Banker et al. (1998), who apply a similar model on the impact of different dimensions of competition.

15 Ritz (2014, p. 171, n. 10) cites computer parts, and metals, cement or crude oil for which there exist different quality grades, or some financial services as examples of differentiated outputs that can be treated as additive.

16 The two first terms in (18) correspond to $\sum_{i=1}^{n} a_{i} y_{i}$ in Ritz (2014), who assumed exogenous quality.
} 


$$
p_{i}=a+k q_{i}-b y=a+k q_{i}-b \sum_{i=1}^{n} y_{i} .
$$

The willingness to pay is now increasing in $q_{i}$ also when all firms provide the same increased quality, in contrast to Sects. 2, 3.

First, consider case (a). The profits of firm $i$ are then:

$$
\pi_{i}=\left(a+k q_{i}\right) y_{i}-b y y_{i}-c y_{i}-\frac{1}{2} q_{i}^{2} .
$$

We focus on two-stage maximisation; we refer to "Appendix 11" for the case of simultaneous maximisation. Maximise (20) with respect to $y_{i}$, given $y_{j}, j \neq i$ to get:

$$
y_{i}\left(q_{i}\right)=\frac{a+k q_{i}-b y-c}{b},
$$

There are $n$ such expressions; add them and rearrange to get aggregate output:

$$
y=\frac{n(a-c)+k \sum_{j=1}^{n} q_{i}}{b(n+1)} .
$$

Insert (22) and (21) into (20), after using (22) to eliminate $y$ from (21):

$$
\pi_{i}\left(q_{i}\right)=\frac{\left(a+n k q_{i}-k \sum_{j \neq 1}^{n} q_{j}-c\right)^{2}}{b(n+1)^{2}}-\frac{1}{2} q_{i}^{2} .
$$

Maximise (23) with respect to $q_{i}$ and note that the assumption $2 b>k^{2}$ ensures concavity and positive solutions. Impose symmetry, solve for $q^{C}$, and insert the solution into (21):

$$
\begin{aligned}
y^{C} & =\frac{n(n+1)(a-c)}{b(n+1)^{2}-2 n k^{2}}, \\
q^{C} & =\frac{2 k n(a-c)}{b(n+1)^{2}-2 n k^{2}} .
\end{aligned}
$$

To compare (24)-(25) to the social optimum, maximise $y$ when $n=1$ under the break-even constraint $a y+k q y-b y^{2}-c y-q^{2} / 2=0 \quad$ (because $p y=a y+k q y-b y^{2}$, which also means that consumer utility becomes $u^{G}=\left(b y^{2} /\right.$ 2 ) $+m$ ). We get an optimal solution whenwver $2 b>k^{2}$ (see “Appendix 12"):

$$
\begin{aligned}
y^{G} & =\frac{2(a-c)}{2 b-k^{2}}, \\
q^{G} & =\frac{2 k(a-c)}{2 b-k^{2}} .
\end{aligned}
$$

We can now make the following observations: 
(1) Like in Sect. 3 (except for when $n=2$ ), $y^{C}$ is increasing and $q^{C}$ decreasing in $n$. This also applies to simultaneous maximisation; see "Appendix 12".

(2) A higher $k$ leads to a higher $y^{C}$, unlike in Sect. 3, where a stronger preference for quality as reflected in $\rho$ had no similar impact.

(3) Simultaneous maximisation yields lower values of $y^{C}$ and $q^{C}$ than in (24)(25) if $n>1$ (see "Appendix 12"); as for a comparison to Ma and Burgess (1993), see Sect. 4.1.

(4) Profits are decreasing in $n$; there is an upper limit for the number of firms for some values of $\beta$, but not when $\beta=2$.

(5) Output and quality are too low, but unit costs are lower in the oligopoly than in the socially optimal allocation (see "Appendix 13", and "Appendix 12" on simultaneous maximisation).

Observations (1)-(3) follow directly from (24)-(25). As for (4), profits per firm are

$$
\pi_{i}=b\left(\frac{y^{C}}{n}\right)^{2}-\frac{1}{2}\left(q^{C}\right)^{2}=\frac{(a-c)^{2}\left[b(n+1)^{2}-2 n^{2} k^{2}\right]}{\left[b(n+1)^{2}-2 n k^{2}\right]^{2}}
$$

so $\partial \pi_{i} / \partial n<0$. The absence of an upper limit to $n$ follows from the fact that $\pi_{i}=0$ would require $n^{2} /(n+1)^{2}=b / 2 k^{2}$, which would violate the condition $2 b>k^{2}$. $^{17}$

The fact that utility $u$ is $b y^{2} / 2+m$ under both types of allocations means that $y^{G} \geq y^{C} \Leftrightarrow u^{G} \geq u^{C}$. As for the total surplus (TS), we get $T S^{G}=u^{G}$ and $T S^{C}=u^{C}+\sum_{i} \pi_{i}$ :

$$
\begin{gathered}
T S^{G}=u^{G}=\frac{b[2(a-c)]^{2}}{2\left(2 b-k^{2}\right)^{2}}+m, \\
T S^{C}=\frac{\left[b n(n+1)^{2}(n+2)-4 k^{2} n^{3}\right](a-c)^{2}}{2\left[b(n+1)^{2}-2 n k^{2}\right]^{2}}+m .
\end{gathered}
$$

Differentiate to see that $d T S^{C} / d n>0$ and note that the condition $2 b>k^{2}$ implies that $T S^{G}>\lim _{n \rightarrow \infty} T S^{C}=(a-c)^{2} /(2 b)+m$. It follows from (25) that a very high $n$ means a zero quality rather than a convergence towards social optimality.

Next, suppose that the total costs are $\left(c_{0}+0.5 q_{i}^{2}\right) y_{i}$, so that quality is reflected only in marginal costs:

$$
y^{C}=\frac{n\left(a-c_{0}+k^{2} / 2\right)}{b(n+1)},
$$

\footnotetext{
${ }^{17}$ However, there may exist an upper limit to the $n$ under more general conditions, as illustrated by the example $b=4, a-c=10, k=s=1$, and $\beta=4$. The highest number of firms that can break even is then 82 .
} 


$$
y^{G}=\frac{a-c_{0}+k^{2} / 2}{b},
$$

This implies that $q^{C}=q^{G}=k$, so there is no under-provision of quality. Also, the number of firms has no impact. These observations conform to Sect. 3 .

\section{Discussion and concluding remarks}

The impact of the number of firms depends on how quality affects different types of costs. The presence of quality-related sunk costs means that quality in an oligopoly is below its socially optimal level and decreasing in the number of firms except for when it increases from two to three. While the number of firms is restricted on such a market, the quality reduction caused by entry (when possible) cannot be dismissed as exceptional. In such cases, an oligopoly usually means a combination of too low quality and too low output, but it turns out that firms may also under some circumstances produce too much of a cheap low-quality output. However, quality is socially optimal and independent on the number of firms if only marginal costs are affected. Moreover, increased competition in the sense of a larger market would not necessarily have an adverse impact on quality despite a larger number of firms.

We have limited ourselves to Cournot-competition and ex-post homogeneous goods, because such markets would normally approach the socially optimal solution when the number of firms becomes sufficiently high. The fact that this is not the case when there are quality-related sunk costs, and no additional market failures such as product differentiation, is an important finding. However, similar results on low quality, a negative impact of entry, and a positive impact of market size can be found in the literature on differentiated goods and Bertrand-competition (see Sect. 1).

The need to analyse these topics (also) through theoretical modelling is highlighted by difficulties in measuring welfare and quality. However, that several empirical studies have assessed market performance after the introduction of competition and profit maximisation. For example, while consumer prices were often reduced in the electricity industry, it appears as less reliable, partly because of reduced infrastructure investments (Lieb-Dóczy et al. 2003; Brunekreeft and Keller 2000; Moss 2004; Jiang and Yu 2004). Similar concerns have been raised about reliability and even safety when it comes to railway liberalisation (Crompton and Jupe 2003; Newbery 2006).

As for the socially optimal solution, more research is needed on how it should be defined when there are quality-related sunk costs. Also, there is no consensus on whether social optimality should be seen as just a point of comparison, or as a realistic alternative that can be achieved under public ownership (or under nonprofit maximising private ownership) ${ }^{18}$ For example, inability or insufficient incentives can in principle cause failures that overshadow the failures related to

\footnotetext{
${ }^{18}$ Moreover, those who manage real-world state-owned enterprises are often required to emphasise profitability rather than social welfare. This might be the case in particular after public-sector reforms that lead to corporatisation (as for example suggested by the New Public Management; see Evans et al., 2005).
} 
market provision. Whether this tends to be the case in reality is partly an open question; the theoretical and empirical literature has tended to yield mixed results. However, there seems to be no universal rule about the superiority of a profitmaximising oligopoly to a welfare-maximising monopoly (for analysis and surveys, see Willner 2001; Willner and Parker 2007; Grönblom and Willner 2014). More research is therefore needed also on the normative implications of the mechanisms that we have studied. Moreover, some firm owners and employees may be partly driven by non-pecuniary motives, at least in industries where quality matters. ${ }^{19}$

Acknowledgements Open access funding provided by Abo Akademi University (ABO). Part of the work was made when the authors were visiting University of Warwick in 2018. Constructive comments to a previous version have been provided by participants in our Department's research seminar, in the $X X X V I I$ Annual Meeting of the Finnish Economic Association, in a seminar arranged by Aboa Centre for Economics, by Hannes Kuch, and by the Editor and two anonymous reviewers. Niklas Grönblom has provided valuable research assistance. A grant from the Krogius foundation (Svenska Litteratursällskapet) is gratefully acknowledged by Sonja Grönblom.

Open Access This article is licensed under a Creative Commons Attribution 4.0 International License, which permits use, sharing, adaptation, distribution and reproduction in any medium or format, as long as you give appropriate credit to the original author(s) and the source, provide a link to the Creative Commons licence, and indicate if changes were made. The images or other third party material in this article are included in the article's Creative Commons licence, unless indicated otherwise in a credit line to the material. If material is not included in the article's Creative Commons licence and your intended use is not permitted by statutory regulation or exceeds the permitted use, you will need to obtain permission directly from the copyright holder. To view a copy of this licence, visit http:// creativecommons.org/licenses/by/4.0/.

\section{Appendices}

\section{Appendix 1: Deriving demand and the equality $q_{i}^{\rho} / p_{i}=q_{j}^{\rho} / p_{j}$}

Consider an individual $h$ who consumes the output of the $i$ :th firm, $i=1,2, \ldots n$. Utility maximisation under the constraint that consumption adds up to the income $m$ means maximising the following Lagrange function:

$$
\max _{y, y_{0}} L=\left(y_{h i} q_{i}^{\rho}\right)^{\alpha_{1}} z_{h}^{\alpha_{0}}+\lambda\left(m-p_{i} y_{h i}-z_{h}\right) .
$$

Each individual then demands

$$
y_{h i}=\frac{\alpha_{1} m}{p_{i}}
$$

\footnotetext{
19 There may on the other hand be a tendency for pecuniary motives to become more dominant. For example, ownership in the maritime industry in Finland used to be dominated by former sea captains and other persons with an emotional attitude to shipping but is now becoming increasingly dominated by investors who are mainly interested in profitability (Harald 2016).
} 


$$
y_{0}=\alpha_{0} m \text {. }
$$

To understand the equality $q_{i}^{\rho} / p_{i}=q_{j}^{\rho} / p_{j}$, insert (35) and (36) into (34) to get the indirect utility function. The utility level is then proportional to

$$
\left(\frac{q_{i}^{\rho}}{p_{i}}\right)^{\alpha 1}
$$

This is the only component in the indirect utility function that depends on which firm that produces $y$. All customers are identical utility maximisers, so the following must hold true for every firm $j$ and $i$ in the industry:

$$
\frac{q_{i}^{\rho}}{p_{i}}=\frac{q_{j}^{\rho}}{p_{j}} .
$$

This implies $p_{j}=\left(q_{j} / q_{i}\right)^{\rho} p_{i}$ for all $i$ and $j$.

\section{Appendix 2: Deriving the Cournot-equilibrium}

Maximise the profit function (5) with respect to $y_{1}$ :

$$
\frac{R}{y_{1}+\sum_{j=2}^{n} y_{j}\left(q_{j} / q_{1}\right)^{\rho}}-\frac{R}{\left[y_{1}+\sum_{j=2}^{n} y_{j}\left(q_{j} / q_{1}\right)^{\rho}\right]^{2}} y_{1}-c_{0}-q_{1}^{\gamma}=0 .
$$

To see why the profit function is concave, rearrange the second derivative as follows:

$$
\frac{\partial_{\pi i}^{2}}{\partial y_{1}^{2}}=\frac{2 R \sum_{j=2}^{n} y_{j}\left(q_{j} / q_{1}\right)^{\rho}}{\left[y_{1}+\sum_{j=2}^{n} y_{j}\left(q_{j} / q_{1}\right)^{\rho}\right]^{3}} .
$$

This expression is negative, so the profit function is concave in $y_{1}$.

Consider the profit function of another firm $j$, such that $j \neq i$ :

$$
\pi_{j}=\frac{R}{y_{j}+\sum_{i \neq j}^{n} y_{i}\left(q_{i} / q_{j}\right)} y_{j}-\left(c_{0}+q_{j}^{\gamma}\right) y_{j}-s q_{j}^{\beta} .
$$

Its first-order condition is:

$$
\frac{R}{y_{j}+\sum_{i \neq j}^{n} y_{i}\left(q_{i} / q_{j}\right)^{\rho}}-\frac{R}{\left[y_{j}+\sum_{i \neq j}^{n} y_{i}\left(q_{i} / q_{j}\right)^{\rho}\right]^{2}} y_{j}-c_{0}-q_{j}^{\beta}=0 .
$$

Divide (42) by $\left(q_{j} / q_{1}\right)^{\rho}$ (which is rearranged as $\left(q_{j} / q_{1}\right)^{2 \rho} /\left(q_{j} / q_{1}\right)^{\rho}$ in the case of the second term): 


$$
\begin{aligned}
& \frac{R}{y_{j}\left(q_{j} / q_{1}\right)^{\rho}+\sum_{i \neq j}^{n} y_{i}\left(q_{i} / q_{1}\right)^{\rho}} \\
& \quad-\frac{R}{\left[y_{j}\left(q_{j} / q_{1}\right)^{\rho}+\sum_{i \neq j}^{n} y_{i}\left(q_{i} / q_{1}\right)^{\rho}\right]^{2}} y_{j}\left(q_{j} / q_{1}\right)^{\rho}-c_{0}\left(q_{1} / q_{j}\right)^{\rho}-q_{j}^{\gamma}\left(q_{1} / q_{j}\right)^{\rho}=0 .
\end{aligned}
$$

Note that one of the terms in $\sum_{i \neq j}^{n} y_{i}\left(q_{i} / q_{1}\right)^{\rho}$ must equal $y_{1}$, so the denominators of (43) are in fact the same as in (39). Add (39) to the sum of the $n-1$ expressions represented by (43):

$$
\frac{(n-1) R}{y_{1}+\sum_{j=2}^{n} y_{j}\left(q_{j} / q_{1}\right)^{\rho}}-\left[c_{0}\left(1+q_{1}^{\rho} \sum_{j=2}^{n} q_{j}^{-\rho}\right)+q_{1}^{\gamma}+q_{1}^{\rho} \sum_{j=2}^{n} q_{j}^{\gamma-\rho}\right]=0 .
$$

This implies:

$$
y_{1}+\sum_{j=2}^{n} y_{j}\left(q_{j} / q_{1}\right)^{\rho}=\frac{(n-1) R}{\left[c_{0}\left(1+q_{1}^{\rho} \sum_{j=2}^{n} q_{j}^{-\rho}\right)+q_{1}^{\gamma}+q_{1}^{\rho} \sum_{j=2}^{n} q_{j}^{\gamma-\rho}\right]} .
$$

Combine (45) and (39) to get $y_{1}$ as a function of the quality levels:

$$
y_{1}=\frac{(n-1) R\left[c_{0}\left(2-n+q_{1}^{\rho} \sum_{j=2}^{n} q_{j}^{-\rho}\right)+(2-n) q_{1}^{\gamma}+q_{1}^{\rho} \sum_{j=2}^{n} q_{j}^{\gamma-\rho}\right]}{\left[c_{0}\left(1+q_{1}^{\rho} \sum_{j=2}^{n} q_{j}^{-\rho}\right)+q_{1}^{\gamma}+q_{1}^{\rho} \sum_{j=2}^{n} q_{j}^{\gamma-\rho}\right]^{2}} .
$$

Insert (45) and (46) into (5) to get the maximum profits as a function of $q_{1}$ and rearrange:

$$
\pi_{1}=\frac{R\left[c_{0}\left(2-n+q_{1}^{\rho} \sum_{j=2}^{n} q_{j}^{-\rho}\right)+(2-n) q_{1}^{\gamma}+q_{1}^{\rho} \sum_{j=2}^{n} q_{j}^{\gamma-\rho}\right]^{2}}{\left[c_{0}\left(1+q_{1}^{\rho} \sum_{j=2}^{n} q_{j}^{-\rho}\right)+q_{1}^{\gamma}+q_{1}^{\rho} \sum_{j=2}^{n} q_{j}^{\gamma-\rho}\right]^{2}}-s q_{1}^{\beta} .
$$

Consider the first-order condition for an optimal quality level. Set the derivative with respect to $q_{1}$ of (47) equal to zero, using the abbreviation $B$ for $c_{0} \sum_{j=2}^{n} q_{j}^{-\rho}+\sum_{j=2}^{n} q_{j}^{-\rho}$ :

$$
\begin{aligned}
\frac{\partial \pi_{1}}{\partial q_{1}} & =\frac{2 R B(n-1) q_{1}^{\rho-1}\left[c_{0}(2-n)+(2-n) q_{1}^{\gamma}+B q_{1}^{\rho}\right]\left[c_{0} \rho-(\gamma-\rho) q_{1}^{\gamma}\right]}{\left(c_{0}+q_{1}^{\gamma}+B q_{1}^{\rho}\right)^{3}}-\beta s q_{1}^{\beta-1} \\
& =0 .
\end{aligned}
$$

Impose ex post symmetry and multiply the first-order condition by $q$ : 


$$
\frac{2 R(n-1)^{2}\left[c_{0} \rho-(\gamma-\rho) q^{\gamma}\right]}{n^{3}\left(c_{0}+q^{\gamma}\right)}-\beta s q^{\beta}=0 .
$$

To check that the solution of (49) represents a maximum, differentiate (48), impose ex post symmetry, use (49) to replace $\beta s q^{\beta}$, and rearrange. The second derivative of the profit function then becomes:

$$
\begin{aligned}
\left.\frac{\partial^{2} \pi_{1}}{\partial q_{1}^{2}}\right|_{q_{j}=q_{1} \forall j}= & \frac{2 R(n-1)^{2}}{n^{4}\left(c_{0}+q^{\gamma}\right)} \times\left\{\left[c_{0} \rho+(\gamma-\rho) q^{\gamma}\right]\right. \\
& \times\left[-\left(n^{2}-2 n+3\right) \gamma q^{\gamma}-((n-2) \rho+\beta n)\left(c_{0}+q^{\gamma}\right)\right] \\
& \left.-n \gamma(\gamma-\rho)\left(c_{0}+q^{\gamma}\right) q^{\gamma}\right\} .
\end{aligned}
$$

Note that the expression $c_{0} \rho+(\gamma-\rho) q^{\gamma}$ is nonnegative if $\beta \geq 0$, that $\gamma>\rho$, and that $n \geq 2$. The second derivative is therefore unambiguously negative in the symmetric equilibrium.

Rearrange (49) to

$$
2 R(n-1)^{2} c_{0} \rho+2 R(n-1)^{2}(\gamma-\rho) q^{\gamma}-n^{3} c_{0} \beta s q^{\beta}-n^{3} s q^{\beta+\gamma}=0 .
$$

This expression is monotone and decreasing in $q$, so there can be only one real solution. Impose symmetry on (46) and multiply by $n$ to get market output as a function of quality as $y=(n-1) R /\left[n\left(c_{0}+q^{\gamma}\right)\right]$. Combining this with (51) would yield $y^{C}$ and $q^{C}$ for case (c).

We get explicit solutions when cost functions are either of type (a) or of type (b). In case (a) we get $c_{i}=c=1+c_{0}$. Solve for $y$ and $q$ to get (6) and (7). Next, suppose that $s=0$. A similar procedure yields (8) and (9) for case (b).

\section{Appendix 3: Deriving the socially optimal solution}

(i) The break-even constraint yields the following output as a function of quality in case (c):

$$
y^{G}(q)=\frac{R-s q^{\beta}}{c_{0}+q^{\gamma}} .
$$

The socially optimal solution is obtained by maximising $y^{G}(q) q^{\rho}$ with respect to $q$. Multiply the first-order condition by $\left(c_{0}+q^{\gamma}\right)^{2} q^{1-\rho}$ and rearrange:

$$
R c_{0} \rho-R(\gamma-\rho) q^{\gamma}-c_{0}(\beta+\rho) s q^{\beta}-(\beta+\rho-\gamma) s q^{\beta+\gamma}=0 .
$$

Assumption 1(iii) ensures that (53) is monotone and positive for low values of $q$ and negative for high values, so a unique maximum exists. Combining (52) and (53) yields $y^{G}$ and $q^{G}$. The solutions for the special cases (a) and (b), i.e. (11)-(14), are reached by setting $\gamma=0$ (so that $c=1+c_{0}$ ) and $s=0$ respectively. 


\section{Appendix 4: The comparative statics of the socially optimal solution in Sect. 2.3}

We can make the following observations:

(i) An increase in market size means a higher output in cases (a) and (b), and a higher quality in case (a), but has no impact on quality in case (b);

(ii) Output is increasing in the parameters relating costs to quality $(s, \beta$, and $\gamma)$, and decreasing in the preference for quality $(\rho)$ in both cases;

(iii) Quality is increasing in $\rho$, and decreasing in the parameters relating costs to quality $(\beta$ and $\gamma)$, except for when $s(1+\varepsilon) e^{-\varepsilon /(1+\varepsilon)} \geq R$ in case (a) and when $f(\eta) \geq(\eta-1) e^{-\eta /(\eta-1)}>c_{0}$ in case (b). (Note that $e$ stands for Euler's number; $e \approx 2.7183$.)

The fact that a higher $R$ increases the socially optimal output is trivial; as for quality, the break-even constraint implies that a higher $R$ makes it easier to afford higher quality. The impact of $\rho$ and $s$ are also obvious. The ambiguity of the impact of $\beta$ and $\gamma$ needs some elaboration. Consider case (a). Differentiating (12) with respect to $\beta$ yields $\partial q / \partial \beta=[-\ln (R / s)+\ln (1+\varepsilon)-/ \varepsilon(1+\varepsilon)][(R / s) /(1+\varepsilon)]^{1 / \beta} /$ $\beta^{2}$. It follows that the sign of the derivative is the same as the sign of $(1+\varepsilon) e^{-\varepsilon /(1+\varepsilon)}-R / s$. The function $f(\varepsilon)=(1+\varepsilon) e^{-/ \varepsilon(1+\varepsilon)}$ is increasing in $\varepsilon$, so the derivative is negative for small values of $\varepsilon$, and positive for large values. Develop a second-order Taylor-approximation in terms of the two first derivatives at some point $\varepsilon_{0}$. As $\varepsilon_{0}$ approaches infinity, we get $f(\varepsilon) \approx(2+\varepsilon) / e$; higher order terms vanish. The approximation is very close in the relevant area where $\varepsilon \geq 8 / 3$, and in particular where $R / s$ is large in the intersection point $f(\varepsilon)=R / s$, as can be expected. Thus, the socially optimal quality is decreasing in $\varepsilon$ until $\varepsilon \geq(R / s) e-2$, or $\beta \geq[(R / s) e-2] \rho$.

For example, $R=100, \rho=1$ and $s=1$ would imply that the lowest quality level (0.996) occurs when $\beta \approx 269.828$. The limit of $q^{G}$ as $\beta$ approaches infinity is 1.0 , so quality remains in fact more or less unchanged after reaching its minimum level. Graphical simulations suggest that $q^{G}$ is L- rather than U-shaped.

As for case (b), a similar argument shows that the socially optimal quality is increasing in $\gamma$ if $(\gamma-\rho) e^{-\gamma /(\gamma-\rho)}>\rho c_{0}$ and vice versa. Denote $\gamma / \rho$ by $\eta$, so that the condition becomes $f(\eta)=(\eta-1) e^{-\eta /(1-\eta)}>c_{0}$. A similar procedure as above shows that a reasonable approximation of $f(\eta)$ is given by $[-2+\eta] / e$, so that quality is increasing when $\gamma>\left(2+c_{0} e\right) \rho$. However, this approximation works badly close to the origin. A second-order Taylor-approximation in $\eta=4$ then works better; we then get $f(\eta) \approx\left(110+64 \eta+\eta^{2}\right) / 54 e^{4 / 3}$. This means that quality is increasing in $\eta$ when $\eta>\sqrt{1134+304.8581 c_{0}}-32 .^{20}$

For example, $c_{0}=2$ and $\rho=1$ would suggest that $q^{G}$ is decreasing in $\gamma$ as long as $\gamma$ is no larger than 7.290. The optimal quality looks L-shaped when we look at large variations in $\gamma$ and $q^{G}$, and it tends to 1.0 as $\gamma$ approaches infinity. However, $q^{G}$

\footnotetext{
${ }^{20}$ A similar procedure as in case a) would yield $f(\eta) \approx[-2+\eta] / e$. Quality would in other words be increasing when $\gamma>\left(2+c_{0} e\right) \rho$. However, this approximation works only if $c_{0}>2$.
} 
becomes increasing for reasonable values of $\gamma$, and the minimum value of $q^{G}(0.855)$ is less close to unity than in case (a). This suggests that the ambiguity of the impact of $\gamma$ cannot be ignored.

\section{Appendix 5: Proof of Lemma 1}

(i) Inserting (7) into (47) after imposing symmetry and solving for $n$ when profits are zero shows that firms can break even for numbers of firm between the values of $n$ that satisfy the equation

$$
-2 n^{2}+(4+\varepsilon) n-2=0 .
$$

The roots of (54) are:

$$
n_{1,2}=1+\frac{\varepsilon}{4} \pm \sqrt{\frac{\varepsilon^{2}}{16}+\frac{\varepsilon}{2}} .
$$

Note that the smaller root $n_{2}$ violates the condition $n \geq 2$. If $n_{2} \geq 2$, the following inequality would hold true:

$$
-1+\frac{\varepsilon}{4}>\sqrt{\frac{\varepsilon^{2}}{16}+\frac{\varepsilon}{2}} .
$$

It is obvious that this cannot be the case if $\varepsilon \leq 4$. In the opposite case, (56) implies

$$
\left(-1+\frac{\varepsilon}{4}\right)^{2}>\frac{\varepsilon^{2}}{16}+\frac{\varepsilon}{2}
$$

which cannot be true unless $\varepsilon<1$, in contradiction to $\varepsilon>4$. It follows that the number of firms must be an integer value in the interval $[2, \hat{n}]$.

(ii) It follows from (15) that $\hat{n}$ is increasing in $\varepsilon$, so the definition of $\varepsilon$ implies that $\hat{n}$ is increasing in $\beta$ and decreasing in $\rho$. As for the absence of an impact of $R$, the result follows directly from (15).

(iii) Profits per firm are $R / n^{2}$, as follows from (8)-(9) and the fact that $p^{C}=n \gamma c_{0} /$ $(n-1)(\gamma-\rho)$. They are never zero for a finite $n$. QED

\section{Appendix 6: Proof of Proposition 1}

The results for cases (a) and (b) follow directly from (6) and (7); the impact of $n$ in case (a) is obvious from the fact that the derivative of $(n-1)^{2} / n^{3}$ is $(n-1)(3-n) / n^{4}$. As for case (c), implicit differentiation of (49) yields:

$$
\frac{\partial q}{\partial R}=\frac{2(n-1)^{2}\left[\rho c_{0}-(\gamma-\rho) q^{\gamma}\right] /\left[s \beta n^{3}\left(c_{0}+q^{\gamma}\right)\right]}{\left[2 R(n-1)^{2} \gamma^{2} q^{\gamma-1} /\left[n^{3}\left(c_{0}+q^{\gamma}\right)^{2}\right]\right]+\beta^{2} s q^{\beta-1}},
$$




$$
\frac{\partial q}{\partial n}=\frac{\left\{2 R(n-1)(n-3)\left[\rho c_{0}-(\gamma-\rho) q^{\gamma}\right]\right\} /\left[n^{4}\left(c_{0}+q^{\gamma}\right)\right]}{\left[2 R(n-1)^{2} \gamma^{2} q^{\gamma-1} /\left[n^{3}\left(c_{0}+q^{\gamma}\right)^{2}\right]\right]+\beta^{2} s q^{\beta-1}} .
$$

It is obvious that the denominators of both expressions are positive. Both numerators are proportional to the left term in (49), which is positive. It follows that $\partial q / \partial R$ is always positive and that $\partial q / \partial n$ has the same sign as $n-3$. QED

\section{Appendix 7: Proof of Corollary 1}

(i) Take the total differential $\Delta q^{C}$ of (7) when both $n$ and $R$ change, and rearrange the condition for a positive sign to get the result $d R / R_{0}>\left[\left(n_{0}-3\right) /\left(n_{0}-1\right)\right] d n / n_{0}$. (ii) Suppose that $d R / R_{0}=d n / n_{0}$. The condition for a positive sign is then satisfied when $n>1$ as assumed. (iii) The upper limit $\hat{n}$ will be the same in all markets, as follows from Proposition 1. There cannot be more than $\hat{n}$ firms in the integrated market, so $d n / n_{0}=0$. Quality will then increase, as follows from part (ii).QED

\section{Appendix 8: Proof of Proposition 2}

(i) Consumer welfare depends on $N\left(q^{\rho} y / N\right)^{\alpha_{1}}$, but we can maximise $q^{\rho} y$ under a break-even constraint to get the same result. is affected. In case (a), use (6) and (7) to write $q(n)^{\rho} y(n)$ as

$$
\left[q^{G}(n)\right]^{\rho} y^{G}(n)=\left(\frac{2}{s \varepsilon}\right)^{1 / \varepsilon}\left[\frac{(n-1)^{\varepsilon+2}}{n^{\varepsilon+3}}\right]^{1 / \varepsilon} .
$$

It is obvious that consumer welfare is increasing in $R$. As for the impact of the market structure, differentiate (60):

$$
\frac{\partial\left[q^{G}(n)\right]^{\rho} y^{G}(n)}{\partial n}=\left(\frac{2}{s}\right)^{1 / \varepsilon} \frac{R^{(\varepsilon+1) / \varepsilon}}{c}\left[\frac{(n-1)^{\varepsilon+2}}{n^{\varepsilon+3}}\right]^{(1-\varepsilon) / \varepsilon} \frac{(n-1)^{1+\varepsilon}(3+\varepsilon-n)}{n^{\varepsilon+4}} .
$$

This expression is strictly positive for $n<\varepsilon+3$ and vice versa. However, firms cannot break even if $n \approx \varepsilon+3$, because (15) would then imply

$$
4+\varepsilon+\sqrt{\varepsilon^{2}+8 \varepsilon}>4 \varepsilon+12,
$$

or

$$
0>\beta^{2}+5 \beta+8
$$

This cannot be true, because the expression to the right in (63) is positive for all values of $\beta$. It therefore follows that consumer welfare is increasing in $n$ in the feasible interval. As for case (b), output is increasing in both $R$ and $n$ according to (8), whereas it follows from (9) that $q$ can be treated as a constant in $\left(q^{\rho} y / N\right)^{\alpha_{1}}$. We 
conclude that consumer welfare is increasing in both $R$ and $n$. Part (i) is thereby proved.

(ii) Consider first case (c). The break-even constraint ensures that the socially optimal output is

$$
y^{G}(q)=\frac{R-s q^{\beta}}{c_{0}+q^{\gamma}} .
$$

Social optimality means maximising $y^{G}(q) q^{\rho}$ with respect to $q$. Take the firstorder condition, use the abbreviation $T^{G}$ for $\left[\rho c_{0}-(\gamma-\rho)\left(q^{G}\right)^{\gamma}\right] /\left[\beta\left(c_{0}+\left(q^{G}\right)^{\gamma}\right)\right]$, and rearrange:

$$
s\left(q^{G}\right)^{\beta}=\frac{R T^{G}}{1+T^{G}}
$$

As for the $n$-firm oligopoly, rearrange (49) using the abbreviation $T^{P}$ for $\left[\rho c_{0^{-}}\right.$ $\left.(\gamma-\rho)\left(q^{P}\right)^{\gamma}\right] /\left[\beta\left(c_{0}+\left(q^{P}\right)^{\gamma}\right)\right]$ :

$$
s\left(q^{P}\right)^{\beta}=\frac{2(n-1) R T^{P}}{n^{3}} .
$$

We now formulate the antithesis $q^{P} \geq q^{G}$. This would mean that setting $q^{P}$ equal to $q^{G}$ (which would mean $T^{P}=T^{G}=T$ ) would make (66) non-negative. Note that $s\left(q^{P}\right)^{\beta}$ is then the same as in (65). This implies

$$
\frac{2(n-1)^{2}}{n^{3}}-\frac{1}{1+T} \geq 0 \Leftrightarrow T \geq \frac{n^{3}-2 n^{2}+4 n-2}{2(n-1)^{2}} .
$$

We shall show that the antithesis implies that firms cannot break even if $n \geq 2$, so an equilibrium where oligopolists produce too high quality cannot exist. Imposing symmetry in (49) implies that firms are unable to break even unless

$$
\frac{R}{n^{2}} \geq s\left(q^{\rho}\right)^{\beta}=\frac{2 R(n-1)^{2} T^{P}}{n^{3}}
$$

holds true. Rearrange this condition:

$$
\frac{n}{2 R(n-1)^{2}} \geq T
$$

Combine (67) and (68). It is obvious that satisfying both conditions would require

$$
0>n^{3}-2 n^{2}+4 n-2 .
$$

This condition is violated for all $n \geq 2$. This implies that quality is too low in the $n$-firm oligopoly. 
It is obvious from (9) and (14) that quality would not be too low without qualitydependent sunk costs. As for case (a), the highest quality occurs when $n=3$. Insert $n=3$ into (7):

$$
q^{P}(3)=\left(\frac{8 R}{27 \varepsilon}\right)^{1 / \varepsilon}
$$

Suppose as an antithesis that the 3-firm oligopoly offers at least as high quality as the welfare maximising public monopoly. It follows from (12) and (71) that this happens if and only if

$$
\frac{8}{27 \varepsilon} \geq \frac{1}{1+\varepsilon}
$$

However, this would require $\varepsilon \leq 0.421$, and three firms can break even only if $\varepsilon>2.667$. The antithesis is therefore false. It follows from Proposition 1 that $q^{P}(n)$ is even lower if $n=2$ or if $n \geq 4$. This implies that quality is too low in the $n$ firm oligopoly. As for case (b), it is obvious from (8), (13), (9), and (14) that output is too low in the $n$-firm oligopoly, but this does not apply to quality.

(iii) It is obvious from (6), (8), (11), and (13) that the output becomes too low in case (b). As for case (a) it follows from (11) and (6) that $y^{C} \geq y^{G}$ applies when $n \geq 1+\varepsilon$. For this to be possible, more than $1+\varepsilon$ firms should be able to break even:

$$
\hat{n}=1+\frac{\varepsilon}{4}+\sqrt{\frac{\varepsilon^{2}}{16}+\frac{\varepsilon}{2}} \geq 1+\varepsilon .
$$

Rearranging shows that this would imply $1 \geq \varepsilon$. It is obvious that two firms cannot break even if there is strict inequality, so the output is too low except for when $n=1+\varepsilon=2$. As for case (c), the ambiguity of the comparison between the oligopoly and social optimality is demonstrated by our numerical example after the proposition. QED

\section{Appendix 9: Proof of Corollary 2}

(i) Marginal costs $c_{0}+q^{\gamma}$ are in case (c) higher in the socially optimal solution than in the market allocation because $q$ is higher. They are independent of quality in case (a) and hence equal under both regimes, and they are equal in case (b) because of the absence of a quality difference.

(ii) Use (11) and (12) and note that unit costs s ATC are $c+s q^{\beta} / y^{G}$ in the socially optimal allocation in case (a):

$$
A T C^{G}=c\left(1+\frac{1}{\varepsilon}\right)
$$

To get the unit costs $A T C^{C}=c+n s q^{\beta} / y^{C}$ of the $n$-firm oligopoly, use (7) and (6) (after dividing $y^{C}$ by $n$ ): 


$$
A T C^{C}=c\left(1+\frac{2(n-1)}{n \varepsilon}\right) .
$$

Setting $n=2$ yields the same expression as (74), but (75) is increasing in $n$, so any $n>2$ implies higher unit costs than in the socially optimal solution. Unit costs and marginal costs are equal in case (b) and hence the same in both regimes. As for case (c), unit costs are $c_{0}+q^{\gamma}+s q^{\beta} / y^{G}$ and $c_{0}+q^{\gamma}+n s q^{\beta} / y^{P}$ respectively. Marginal costs are lower in the oligopoly, but the average sunk costs may be both higher and lower, because $q^{P}<q^{G}$, and because $q^{P} / n$ is likely to be lower than $q^{G}$. QED

\section{Appendix 10: Proof of Proposition 3}

(i) It follows from (17) and (12) that quality would become too high the oligopoly in case (a) if

$$
\frac{n-1}{\varepsilon n^{3}}>\frac{1}{1+\varepsilon}
$$

Set both sides of the inequality equal and solve for $n$. The oligopoly would overprovide quality in the interval between the solutions

$$
n_{1,2}=\frac{1+\varepsilon}{2 \varepsilon} \pm \sqrt{\left(\frac{1+\varepsilon}{2 \varepsilon}\right)^{2}-\frac{1+\varepsilon}{\varepsilon}} .
$$

but this interval is empty for all $\varepsilon \geq 1 / 3$. Less than two firms would be able to break even if $\varepsilon<1$, so $\varepsilon$ has to be larger than $1 / 3$. This observation rules out the possibility of equal quality or overprovision. As for case (b), the result is obvious from the fact that the allocation is the same as in Proposition 2.

(ii) As follows from (17) and (7), quality is otherwise lower than under two-stage maximisation, but equal when $n=2$.

(iii) Industry output is the same in case (a) as under two-stage maximisation both when it comes to the market allocation and the socially optimal solution. It follows from (6) and (11) that market provision yields the socially optimal output if $n=1+\varepsilon$. However, firms would not break even if $n>1+\varepsilon$, so we can conclude that the output is too low in all other cases. As for case (b), the result is obvious from the fact that the allocation is the same as in Proposition 2. QED

\section{Appendix 11: Simultaneous maximisation under oligopoly in Sect. 4.2}

Simultaneous maximisation of (21) with respect to $y_{i}$ and $q_{i}$ yields:

$$
y^{C}=\frac{n(a-c)}{b n(n+1)-k^{2}},
$$




$$
q^{C}=\frac{k(a-c)}{b n(n+1)-k^{2}} .
$$

It is straightforward to show that (24) always means a higher output than (78). As follows from (25) and (39), quality is higher under simultaneous maximisation in the monopoly case, but otherwise lower. The assumption $2 b>k^{2}$ is sufficient but not necessary for a positive Hessian and for positive solutions, as follows from (78)(79).

\section{Appendix 12: Deriving the socially optimal solution in Sect. 4.2}

It can easily be verified that two-stage maximisation and simultaneous maximisation yield the same result. In the former case, solve for $y$ as a function of $q$, and maximise the consumer surplus with respect to $q$. We then get the same solution when maximising $y$ with respect to $q$. Moreover, the objective function is concave for all values of $k$ and $b$. However, simultaneous maximisation turns out to be more straightforward. Let $\lambda$ stand for the Lagrange multiplier. Maximising consumer utility subject to a break-even constraint can then be expressed as follows:

$$
\max _{y, q} L=\frac{b y^{2}}{2}+m+\lambda\left[a y+k q y-b y^{2}-c y-\frac{1}{2} q^{2}\right] .
$$

Differentiating with respect to $q$ yields $q=k y$. Inserting into the constraint yields $y^{G} ; q^{G}$ is then obtained as $k y^{G}$. This explains (26) and (27). Solve for $\lambda$ from $\partial L / \partial y$. To ensure concavity, calculate $L_{\lambda y}, L_{\lambda q}, L_{y y}$, and $L_{q q}$. Insert the solutions for $y, q$ and $\lambda$. The bordered Hessian then becomes:

$$
|B|=\left[\begin{array}{ccc}
0 & -(a-c) & 0 \\
-(a-c) & \frac{b\left(2 b+k^{2}\right)}{2 b-k^{2}} & \frac{2 b k}{2 b-k^{2}} \\
0 & \frac{2 b k}{2 b-k^{2}} & \frac{-2 b}{2 b-k^{2}}
\end{array}\right] .
$$

The Lagrange function is concave whenever $2 b>k^{2}$ as assumed, in which case the solutions are also positive.

\section{Appendix 13: The comparative statics of Sect. 4.2}

To compare output, consider (24) and (26). Suppose as an antithesis that $y^{C}>y^{G}$. This would imply:

$$
-2 b(n+1)+(3-n) n k^{2}>0 .
$$

This can never be the case if $n \geq 3$. Consider therefore $n=1$ and $n=2$. It follows that both cases would cause (82) to violate the condition $2 b>k^{2}$.

Next, consider quality, and compare (25) to (27). The antithesis $q^{C}>q^{G}$ would imply 


$$
k^{2}>\frac{n^{2}+1}{n} b,
$$

but like in the previous case, this would violate the condition $2 b>k^{2}$.

As for unit costs, note that each firm $i$ has the unit costs $A T C_{i}=c+q^{2} / 2(y / n)$. Compare the Cournot-solution with social optimality:

$$
\begin{gathered}
A T C^{C}=c+\frac{2 k^{2} n^{2}(a-c)}{(n+1)\left[b(n+1)^{2}-2 k^{2} n\right]} . \\
A T C^{G}=c+\frac{2 k^{2}(a-c)}{2\left[2 b-k^{2}\right]} .
\end{gathered}
$$

Suppose as an antithesis that $A T C^{C}>A T C^{G}$. This would imply

$$
k^{2}>\frac{n^{3}-n^{2}+3 n+1}{2 n} b .
$$

The fraction to the right is increasing in $n$ for all $n \geq 1$ Its lowest value is therefore associated with a monopoly. A monopoly would mean $k^{2}>2 b$, so it is obvious that all market structures would imply that (86) violates the assumption $b>2 k^{2}$. The antithesis is therefore false. Unit costs are therefore lower in the Cournot-solution.

\section{References}

Banker RD, Khosla I, Sinha KK (1998) Quality and competition'. Manag Sci 44(9):1179-1192

Belleflamme P, Peitz M (2014) Asymmetric information and overinvestment in quality. Eur Econ Rev 66:127-143

Berry S, Waldfogel J (2010) Product quality and market size. J Ind Econ 58(1):1-31

Brekke KR, Nuscheler R, Straume OR (2006) Quality and location choices under price regulation. J Econ Manag Strategy 15(1):207-227

Brekke KR, Siciliani L, Straume OR (2010) Price and quality in spatial competition. Region Sci Urban Econ 40(6):471-480

Brekke KR, Siciliani L, Straume OR (2011) Hospital competition and cuality with regulated prices. Scand J Econ 113(2):444-469

Brunekreeft G, Keller K (2000) The electricity supply industry in Germany: market power or power of the market? Utility Policy 9:15-29

Carlin W, Soskice D (2006) Macroeconomics: imperfections, institutions and policies. Oxford University Press, Oxford

Choi DY, Stack MH (2005) The all-American beer: a case of inferior standard (taste) prevailing? Bus Horiz 48(1):79-86

Crompton G, Jupe R (2003) Such a silly scheme: the privatisation of Britain's railways 1992-2002. Crit Perspect Account 14:617-645

Economides N (1991) Quality variations in the circular model of variety-differentiated Products. Region Sci Urban Econ 23:235-257

Evans B, Richmond T, Shields J (2005) Structuring neoliberal governance: the nonprofit sector, emerging new models of control and the marketisation of service delivery. Policy Soc 24(1):73-97

Gal-Or E (1983) Quality and quantity competition. Bell J Econ 14(2):590-600

Geroski P (1995) What do we know about entry? Int J Ind Organ 13(4):421-440 
Grönblom S, Willner J (2014) Organisational form and individual motivation: public ownership, privatisation, and fat cats. J Econ Policy Reform 17(3):267-284

Harald P (2016) Rolls-Royce valde finskt samarbete [Rolld-Royce chose Finnish Cooperation]. Hufvudstadsbladet, April 11, p. 10

Jiang JN, Yu J (2004) Reliability in electricity markets: another binding constraint. Electr J 17(5):43-53

Kranton RE (2003) Competition and the incentive to produce high quality. Economica 70(279):385-404

Lieb-Dóczy E, Börner AR, MacKerron G (2003) Who secures the security of supply? European perspectives on security, competition and liability. Electr J 16(10):10-19

Ma CA, Burgess JF (1993) Quality competition, welfare, and regulation. J Econ 58(2):153-173

MacKinnon JB (2016) The L.E.D. quandry: why there's no such thing as "built to last". The New Yorker, July 14

Matsumura T, Matsushima N (2007) Congestion-reducing investments and economic welfare in a Hotelling model. Econ Lett 96(2):161-167

Moss DI (2004) Competition or reliability in electricity? What the coming policy shift means for restructuring. Electr J 17(2):11-28

Newbery DM (2006) Privatising network industries. In: Koethenbürger M, Sinn H-W, Whalley J (eds) Privatization experiences in the EU. MIT Press, Cambridge, pp 3-50

Ritz RA (2014) On welfare losses due to imperfect competition. J Ind Econ 57(1):168-190

Rivera Batiz LA, Romer PM (1991) Economic Integration and Endogenous Growth. Q J Econ 106(2):531-555

Sheshinski E (1976) Price, quality and quantity regulation in monopoly. Economica 43(170):127-137

Spence AM (1975) Monopoly, quality, and regulation. Bell J Econ 6(2):417-429

Sutton J (1991) Sunk costs and market structure. Price competition, advertising and the evolution of concentration. MIT Press, Cambridge

Tirole J (1988) The theory of industrial organization. MIT Press, Cambridge

Vives X (2008) Innovation and Competitive Pressure. J Ind Econ 56(3):419-464

Willner J (2001) Ownership, efficiency, and political interference. Eur J Polit Econ 17(6):723-748

Willner J, Parker D (2007) The performance of public and private enterprise under conditions of active and passive ownership and competition and monopoly. J Econ 90(3):221-253

Publisher's Note Springer Nature remains neutral with regard to jurisdictional claims in published maps and institutional affiliations. 